\title{
Determination of Wave Speed and Distensibility of Flexible Tubes Using Diameter and Velocity
}

\author{
Ye Li, Student Member, IEEE EMBS, Ashraf W. Khir
}

\begin{abstract}
It is well accepted that wave speed is one of the key factors describing wave propagation in arteries [1]. Local wave speed is directly related to the mechanical properties of the arterial wall [2] and is widely used to determine the arterial distensibility [3]. Several methods have been proposed for determining wave speed in arteries, such as foot-to-foot and PU-loop methods. In this paper, we suggest a new method for the determination of wave speed and wall distensibility, using noninvasive measurements. The theoretical foundation of this method is based on the 1-D conservation of mass and momentum equations of flow in flexible tubes. We simultaneously measured pressure, diameter and velocity at the same site, sequentially along silicon and latex tubes which are $1 \mathrm{~m}$ in length and of different diameters. We compared the results of the new method, $\ln (D) U-l o o p$, with those determined by the PU-loop method. Wave speeds determined by both methods are comparable, although wave speeds determined by the new technique are slightly smaller than those determined by PU-loop method. We also compared distensibility calculated by the new method with those calculated using the traditional method $\left(D_{t}\right), D_{t}=d P / A d A$, where $A$ and $d A$ are the cross sectional area and its change respectively, and $\mathrm{dP}$ is the change in pressure. The results of both methods are in agreement. We conclude that the new technique has the advantage of using only noninvasive parameters which is of clinical relevance.
\end{abstract}

\section{INTRODUCTION}

A rterial wave speed, often referred to as the pulse wave velocity by physiologists and clinicians, is the speed at which changes in pressure and velocity travel along the artery. The wave speed depends chiefly upon the local properties of the arterial wall [4]. It is widely used to determine arterial distensibility [3] and has been used as a surrogate marker for cardiovascular disease [5]. Several methods have been proposed to determine wave speed in arteries, the most common way to measure wave speed is foot-to foot method. This method involves the simultaneous measurement of either pressure or velocity at two sites as known distance apart (L) and determining the time delay between the two measurements $(\Delta t)$, so the wave speed $\mathrm{c}=\mathrm{L} / \Delta \mathrm{t}$ [4]. In 2001, Khir developed a PU-loop method to determine the local wave speed; this is a technique for the determination of local wave speed only using measurements of pressure and flow at the

Manuscript received April 7, 2009

Ye Li, Biofluid Mechanics Group, Brunel Institute for Bioengineering, Brunel University, Greater London, UK ( ye.li@ brunel.ac.uk)

Ashraf W. Khir, Biofluid Mechanics Group, Brunel Institute for Bioengineering, Brunel University, Greater London, UK (Ashraf.Khir@brunel.ac.uk) same site [6]. However, most of these methods have one or more drawbacks. The invasive nature of pressure measurement probably is not very suitable for routine examination [7].

Distensibility is directly related to wave speed and used for assessing the mechanical properties of arterial wall. To determine the distensibility of an arterial wall, the pressure and cross sectional area of the artery should be measured simultaneously. Cross sectional area of artery can be obtained relatively accurately by diameter using ultrasound (US) [8] Pressure, again, because of its invasive nature, made the measurement inconvenient. However, the local wave speed in a thin - walled, uniform, flexible vessel containing an incompressible fluid, is related directly to the distensibility via the Bramwell-Hill expression [9].

$$
c= \pm \sqrt{\frac{1}{\rho D s}},
$$

Where $\mathrm{c}$ is the local wave speed, $\rho$ is the density of the fluid, Ds is the segment distensibility.

Based on knowledge of local wave speed and distensibility, we developed a new method to determine the wave speed and distensibility of flexible tube, noninvasively. In this paper, we will present this new method, and also test experimentally the relative accuracy of the results for determining wave speed and distensibility in flexible tubes.

\section{METHODS}

\section{A. Theoretical Methods}

The water hammer equation can be written as

$$
d P_{ \pm}= \pm \rho c d U_{ \pm}
$$

Where $\mathrm{dP}$ and $\mathrm{dU}$ are the changes in pressure and velocity respectively, $\rho$ is density, $\mathrm{c}$ is wave speed and \pm indicates the forward and backward directions. Wave speed, c, is a function of the distensibility of tube wall

$$
c^{2}=\frac{1}{\rho D_{s}}
$$

Where $D_{s}$ is the segment distensibility, $A$ is assumed the circular initial cross section of the vessel

$$
D_{t}=\frac{1}{A} \frac{d A}{d P}
$$

Changes in pressure, $\mathrm{dP}$, can be considered as the linear summation of the change in pressure in the forward (+) and backward (-) direction,

$$
d P=d P_{+}+d P_{-}
$$


It is also reasonable to assume that changes in the vessel diameter, $\mathrm{dD}$, can also be considered as the linear summation of diameter change due to changes in the forward and backward pressure changes,

$$
d D=d D_{+}+d D_{-}
$$

By using the equations above, wave speed can be expressed in terms of $U$ and $D$.

$$
c=\frac{D}{2} \frac{\left(d U_{+}-d U_{-}\right)}{\left.d D_{+}+d D_{-}\right)}
$$

If we assume $\mathrm{D}$ is a constant that equals the unstretched diameter ( $\mathrm{D}_{0}$ in the in-vitro experiments), and further consider only forward waves

$$
c=\frac{D}{2} \frac{d U_{+}}{d D_{+}}
$$

If we consider that $\mathrm{dD} / \mathrm{D}$ which is the incremental hoop stress equals $d \ln (\mathrm{D})$, we can introduce the new expression of wave speed in terms of $U$ and $\ln (\mathrm{D})$

$$
c= \pm \frac{1}{2} \frac{d U_{ \pm}}{d \ln D_{ \pm}}
$$

Eq. (9) describes a linear relationship between $U$ and $\ln (\mathrm{D})$ in the absence of reflections, and if we plot $\ln (\mathrm{D})$ against $U$, we should get a linear portion in the early part of the cycle.

Substituting Eq. (9) into Eq. (3), distensibility can be expressed in terms of diameter and velocity, both of which can be measured noninvasively.

$$
D_{s}=\frac{4}{\rho}\left(\frac{d \ln D_{+}}{d U_{+}}\right)^{2}
$$

\section{B. Experimental Methods}

The general experimental set up used in the study is shown in Fig. 1 and a description of the individual elements follows.

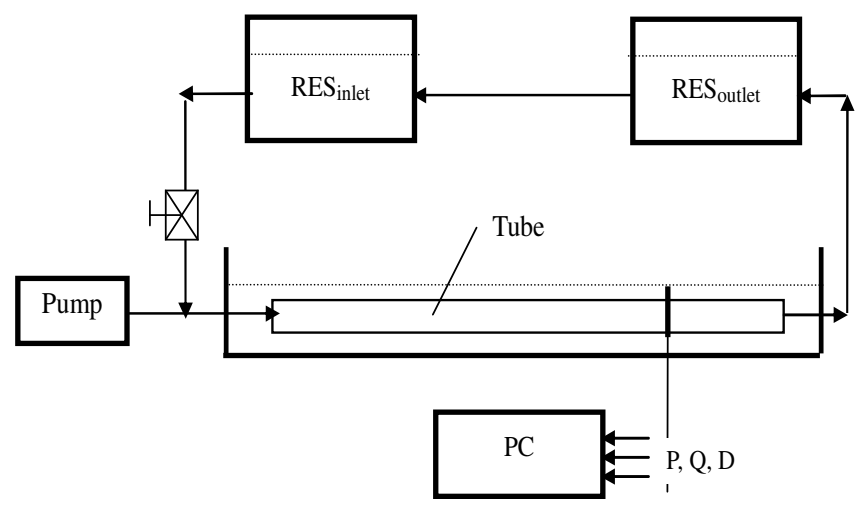

1) Tubes

Fig. 1. A schematic diagram of the experiment set up.

We measured wave speed and distensibility in different materials and sizes of flexible tubes, whose dimensions are given in Table I. Each of the tubes is uniform in both dimension and mechanical properties along its $1 \mathrm{~m}$ in length. The tubes were fully merged into a water tank, where the water level was approximately $1 \mathrm{~cm}$ above the tubes. Each tube was kept in the horizontal position.

2) Pump

The inlet of the tube was connected to a piston pump, which produced an approximately semi-sinusoidal single pulse wave. An 11 Watts graphite brushes DC motor (Maxon 110937, A-max, Sachseln, Switzerland) was used to drive the pump. The motor used a constant DC power supply of 5.6 Volts.

\section{3) Reservoirs}

The inlet and outlet of each tube were connected to the inlet and outlet reservoirs, respectively. The height of the fluid in the reservoirs was adjusted to $10 \mathrm{~cm}$ above the longitudinal axis of the tube; producing an initial hydrostatic pressure of approximately $1 \mathrm{KPa}$. We note that although the transmural pressure for the different-sized tubes will vary, this variation was ignored as it was not significant and its effect was expected to be minimal.

4) Valve

One-way valve was placed between inlet of the tubes and the inlet reservoir as illustrated in Fig. 1. The function of the valve

\begin{tabular}{|c|c|c|c|}
\hline Material & $\begin{array}{l}\text { Unstressed } \\
\text { internal } \\
\text { diameter } \\
(\mathrm{mm})\end{array}$ & $\begin{array}{c}\text { Wall } \\
\text { thickness } \\
(\mathrm{mm})\end{array}$ & $\begin{array}{c}\text { Initial } \\
\text { external } \\
\text { diameter } \\
(\mathrm{mm}) \\
\end{array}$ \\
\hline \multirow{8}{*}{ Silicon } & \multirow{3}{*}{8} & 1 & 10 \\
\hline & & 2 & 12 \\
\hline & & 3 & 14 \\
\hline & \multirow{3}{*}{10} & 1 & 12 \\
\hline & & 2 & 14 \\
\hline & & 3 & 16 \\
\hline & \multirow{2}{*}{16} & 2.4 & 20.8 \\
\hline & & 3 & 22 \\
\hline \multirow{3}{*}{ Latex } & 8.5 & 0.15 & 8.8 \\
\hline & 24.2 & 0.15 & 24.5 \\
\hline & 32.3 & 0.15 & 32.6 \\
\hline
\end{tabular}
was to prevent any portion of the displaced volume of water at inlet flow into the reservoir.

TABLE I

DIMENSIONS OF TUBES

\section{5) Measurements}

Simultaneous pressure, flow and diameter waveforms were measured at the same sites, sequentially in time, every $25 \mathrm{~cm}$ along the tubes which are $1 \mathrm{~m}$ in length each. Pressure and flow were measured using a $8 \mathrm{~F}$ tipped catheter pressure transducer (Millar Instruments Inc., Houston, Texas, USA) and ultrasonic flow probe (Transonic System, Inc, Ithaca, NY, USA), respectively. The external diameters of the different sized tubes were measured using ultrasonic paired crystals (Sonometrics Corporation, London, Ontario, Canada). Wall thicknesses were measured by a caliber. All the data were acquired at a sampling rate of $500 \mathrm{~Hz}$ using Sonolab (Sonometrics Corporation, London, Ontario, Canada). The analysis procedure was carried out using programs written in Matlab (The Mathworks, Natick, MA, USA).

\section{RESULTS}

\section{A. Wave speed}

We tested the relative accuracy of the $\ln (\mathrm{D}) \mathrm{U}$-loop for determining wave speed in our experiments by comparing its 
results to the results of the PU-loop method. Fig. 2 shows the wave speed measured by $\ln (\mathrm{D}) \mathrm{U}-\mathrm{loop}$ in a $10 \mathrm{~mm}$ silicon tube.

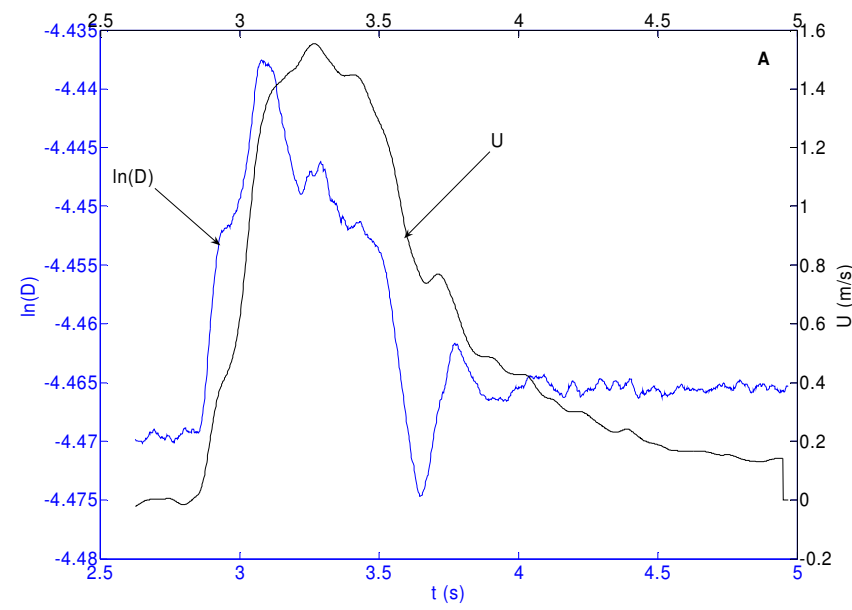

TABLE II

WAVE SPEEDS DETERMINED BY PU-LOOP AND LN(D)U-LOOP

\begin{tabular}{|c|c|c|c|c|c|}
\hline $\begin{array}{c}\text { Mate- } \\
\text { rial }\end{array}$ & $\begin{array}{c}\text { Internal } \\
\text { diameter } \\
(\mathrm{mm})\end{array}$ & $\begin{array}{c}\text { Wall } \\
\text { thickness } \\
(\mathrm{mm})\end{array}$ & $\begin{array}{l}\text { WS_ } \\
\text { PU-loop } \\
(\mathrm{m} / \mathrm{s})\end{array}$ & $\begin{array}{c}\mathrm{WS}_{-} \\
\ln (\mathrm{D}) \mathrm{U} \\
-\mathrm{loop} \\
(\mathrm{m} / \mathrm{s}) \\
\end{array}$ & $\begin{array}{c}\% \\
\text { Change }\end{array}$ \\
\hline \multirow{8}{*}{ Silicon } & \multirow{3}{*}{8} & 1 & 29.23 & 28.69 & -1.85 \\
\hline & & 2 & 31.56 & 31.04 & -1.64 \\
\hline & & 3 & 33.37 & 32.29 & -3.23 \\
\hline & \multirow{3}{*}{10} & 1 & 27.16 & 27.01 & -0.56 \\
\hline & & 2 & 29.88 & 29.31 & -1.92 \\
\hline & & 3 & 31.11 & 30.51 & -1.97 \\
\hline & \multirow{2}{*}{16} & 2.4 & 22.59 & 22.53 & -0.28 \\
\hline & & 3 & 25.51 & 24.45 & -4.16 \\
\hline \multirow{3}{*}{ Latex } & 8.5 & 0.15 & 5.42 & 5.30 & -2.21 \\
\hline & 24.2 & 0.15 & 2.98 & 2.81 & -5.84 \\
\hline & 32.3 & 0.15 & 2.61 & 2.60 & -0.31 \\
\hline
\end{tabular}

* WS_PU-loop is the wave speed determined by PU-loop

WS_ln(D)U-loop is the wave speed determined by $\ln (\mathrm{D}) \mathrm{U}-\mathrm{loop}$

\section{Distensibility}

We also tested the relative accuracy of the $\ln (\mathrm{D}) \mathrm{U}$-loop for determining distensibility in our experiments by comparing its results to the results of the traditional method using pressure and cross sectional area to calculate the distensibility, Equation (4). We tested the method in every tube for three positions $(25,50,75 \mathrm{~cm})$. It shows the results in Fig. 5. The overall difference between both methods is $3.39 \pm 1.8 \%$.

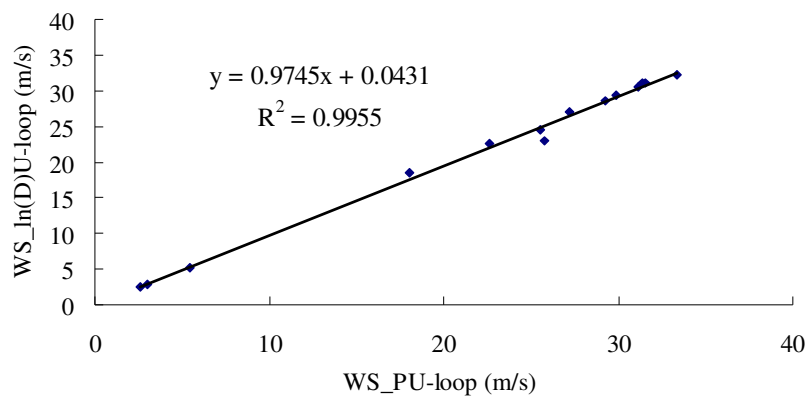

Fig. 3. Correlation of wave speed determined by PU-loop and $\ln (\mathrm{D}) \mathrm{U}-\mathrm{loop}$. The correlation coefficient $\mathrm{R}^{2}=0.9955$.

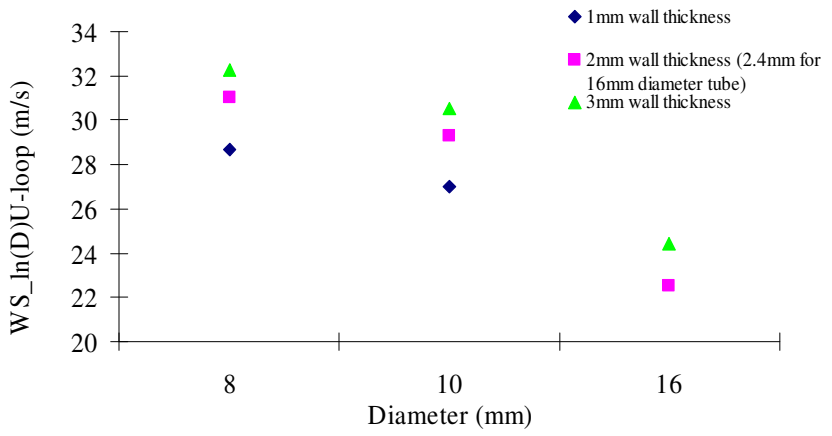

Fig. 4. Wave speed determined by $\operatorname{lnDU}$-loop for different wall thickness of tubes. 

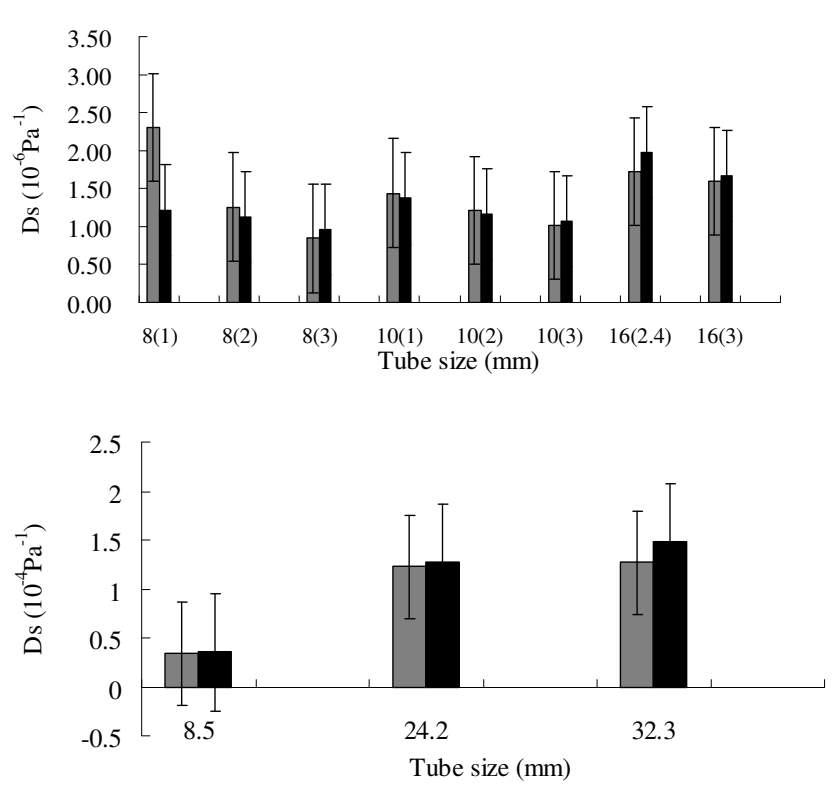

Fig. 5. Distensibility determined by $D_{t}$ (grey) and $\ln (\mathrm{D}) \mathrm{U}-$ loop (black). The top one shows the distensibility of the silicon tubes, the data in brackets are the wall thickness of the tube. The bottom figure shows the distensibility of the latex tubes, all of which had $0.15 \mathrm{~mm}$ wall thickness.

\section{DISCUSSIONS}

As seen in Table I, wave speeds determined by $\ln (\mathrm{D}) \mathrm{U}-\mathrm{loop}$ are very similar to those determined by PU-loop. This could also be seen in Fig. 3, and the wave speeds determined by $\ln (\mathrm{D}) \mathrm{U}$-loop are a little smaller than those determined by PU-loop.

From Fig. 4, we can find out that as the wall thickness increases, the wave speed increases too, this accord with that when the tube wall becomes more rigid, the wave speed in the tube will become greater, but the tube wall will become less flexible, the distensibility will become smaller. So there is no wall thickness required in our method.

As seen in Fig. 5, the distensibilities calculated by Equation (9) are very close to those calculated by $D_{t}$, Equation (4), and the distensibilities of the latex tubes are quite bigger than those of silicon tubes.

It is known that the mechanical properties and dimension in the ascending aorta are dissimilar to those in the peripheral arteries [10]. Also, the wave speed is related to the mechanical properties of the arterial wall. Hence, determination of wave speed using the measurements at one site is very important to hemodynamic study as it can provides direct information about the arterial distensibility. PU-loop method allows for determination of wave speed in one site, but it requires measurements of pressure which is usually obtained invasively. Our method measures velocity and diameter instead of pressure and velocity, needing only the measurement at one site noninvasively.

Our experiments were carried out in flexible tubes, so we use the ultrasound paired crystals to measure the diameter waveforms. In clinical, arterial diameter waveforms are assessed by B-mode (ultrasound), and displayed on the screen of the US scanner, providing a sparse real-time B-mode image [7]. With the ultrasound flow probe measuring blood flow, we could achieve the noninvasive measurements of local wave speed and distensibility.

\section{CONCLUSIONS}

Wave speed and distensibility determined by $\ln (\mathrm{D}) \mathrm{U}$-loop method are very close to those obtained by the PU-loop and $D_{t}$ of determining distensibility respectively. Also the new technique for determining the wave speed is sensitive for different diameter and wall thickness of tubes. The technique has advantages that are clinically relevant; it uses only one measurement site, and it uses measurements of velocity and diameter which can be obtained noninvasively. Also this method is easy to implement, requiring only the determination of the slope of the initial linear part of the $\ln (\mathrm{D}) \mathrm{U}-\mathrm{loop}$.

\section{REFERENCES}

[1] D. A. Mcdonald, Blood flow in arteries, Edward Arnold Ltd, 1998.

[2] W. R. Milnor, Hemodynamics, Williams\&Willkins, 1989.

[3] E. D. Lehmann, K. D. Hopkins, R. G. Gosling, Assessment of arterial distensibility by automatic pulse wave velocity measurement, Hypertension, vol. 27, 1996, pp. 1188-1191.

[4] A. W. Khir, A. Zambanini, K. H. Parker, Local and regional wave speed in the aorta: effects of arterial occlusion, Medical engineering \& Physics, vol. 26, 2004, pp. 23-29.

[5] S. Meaume, A. Rudnichi, A. Lynch, C. Bussy, C. Sebban, A. Benetos et al, Aortic pulse wave velocity as a marker of cardiovascular disease in subjects over 70 years old, Hypertension, vol. 19, 2001, pp.871-877.

[6] A. W. Khir, A. O'brien, J. S. Gibbs, K. H. Parker, Simultaneous determination of wave speed and wave separation in the arteries, Journal of Biomechanics, vol. 34, 2001, pp. 1145-1155.

[7] Jan M. Meinders, Arnold P.G. Hoeks, Simultaneous assessment of diameter and pressure waveforms in the carotid artery, Ultrasound in Med. \& Biol., vol. 30, 2004, pp. 147-154.

[8] A. P. G. Hoeks, P. J. Brands, F. A. Smeets, R. S. Reneman, Assessment of the distensibility of superficial arteries, Ultrasound in Med. \& Biol, vol. 16, 1990, pp. 121-128.

[9] P. J. Brands, J. M. Willigers, L. A. F. Ledoux, R. S. Reneman and A. P. G. Hoeks, 1998, A noninvasive method to estimate pulse wave velocity in arteries locally by means of ultrasound, Ultrasound in Med. \& Biol., vol. 24, pp. 1325-1335.

[10] W. W. Nichols, M. F. O'rourke, McDonald's blood flow in arteries, Theoretical, experimental, and clinical principles, Arnold, 2005. 